\title{
Digital Twins for Simulation Systems
}

\author{
Sudha Rani Jainoju, B. Kishore Babu, T. Annamani, T. Hemalatha
}

\begin{abstract}
Complex Manufacturing Systems can be better engineered with simulation techniques Relaying only on physical system to collect real world and capturing models is out of date. Moreover, a complex system has recursive model design, which leads to consume more time and more maintenance, unplanned down times and poor operating efficiency.

The New Industry 4.0, digital twin creates virtual mirror of actual system. Here we have demonstrated digital twinning of UAV(Parrot ARDrone 2.0). Digital twin is virtual replica of physical assets and it can be simulated with real time data using industrial Internet of Things. Simulation with real time data improves operating efficiency, reduces unplanned down time hence increased revenue to manufactures.
\end{abstract}

\section{Keywords:Digital}

Twin,simulationtechnology, virtualreplica, UAV.

\section{INTRODUCTION}

The progress on miniaturization technologies, with help of embedded control systems, IOT (Sensors), and communication boosted the new development of many new light weight and relatively low cost Unmanned Aerial Vehicles (UAV's). In this contrast constraints towards maintenance of UAV like power consumption weight, size play will play important role in UAV's Performance.with digital twin technology battery, power pay load , avionics paramerters can be closely moniotored which will improve performance of UAV.figure 1 represents the digital twin of aircraft modules.

Digital Twins is a cloud based digital image of an asset it is also called as mind of a machine, it is a process of man mind working with the mind of a machine.it's a living model that drives the outcome of any business updating and learning the nature of its own ,reasoning and optimizing operations to be performed and finally informing to human being and using edge controls it operates.It gets operational data and environmental data and constantly updates itself .It acquires knowledge from the operating machine and learn from them to take decisions and incorporates this into the model and it uses this to drive the business outcomes like predicting failures which reduces maintenance cost and unplanned outages.it also facilitates the optimization of mitigation

Revised Version Manuscript Received on 10 September, 2019.

Sudha Rani Jainoju, Assistant professor,Anurag group of institutions,venkatapur, Andhra Pradesh, India.

(Email: sudharaniece@cvsr.ac.in)

B. Kishore Babu, Assistant professor,Anurag group of institutions, venkatapur, Andhra Pradesh, India.

(Email: annadimpu@gmail.com)

T. Annamani, Assistant professor,Anurag group of institutions,venkatapur, Andhra Pradesh, India.

(Email: annamaniece@cvsr.ac.in)

T. Hemalatha, Assistant professor,Anurag group of institutions, venkatapur, Andhra Pradesh, India.

(Email: themalathaece@cvsr.ac.in) events.its the platform where all the experts brought together providing powerful analysis insight and diagnostics.

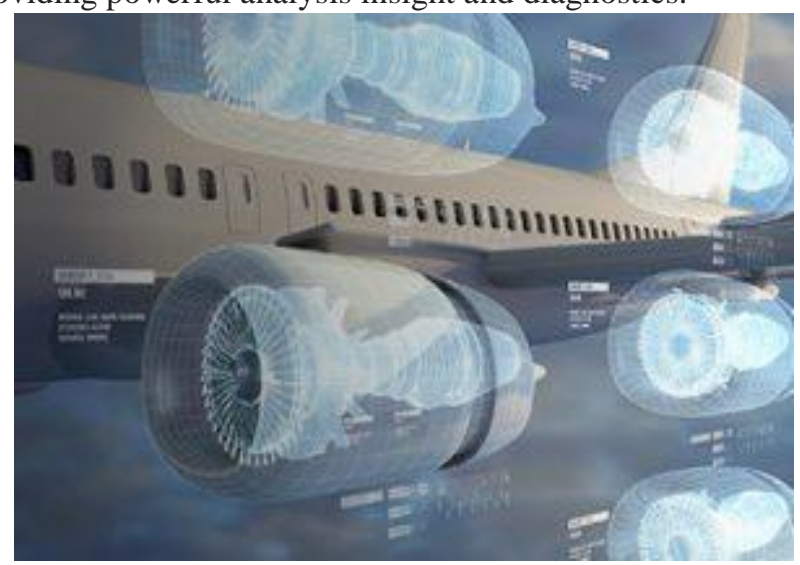

Figure 1:digital twin

Digital twin firstly, gathers the operational data like temperatures, pressures of a machine and environmental data gives to a hybrid model which is combination of physical and digital capabilities', model includes Bayesian learning and machine learning techniques which examine the cumulative data and compare with the other models data and acquire precise conditions of the machine.

Secondly,digital twin does the thinking process by performing over 15000 simulations running on the historical platforms, hybrid models, living models, forecasting models looking at economic factor and cost and analyze solutions and give a number of possibilities with the reasons in two ways either option can be performed manually or an application that's runs with a control system

And finally, In the last stage it informs the human being and executes the operation what is to be done for a situation in a machine.This execution can be done manually or using an application. Using an application it's more convenient to operate and precise minimizations are achieved with $25 \%$ reductions compared with the manual.It is compiled using edge device. Classification of Twins as follows.

\section{Part twins:}

This twin warns about failures of a machine-like rotor failure prediction in a turbine.

\section{Product twins:}

It optimizes the remaining lifetime of any asset which reduces the maintenance cost for e.g.: steam turbine life optimization.

\section{Process twins:}

It maximize the utilization of an asset for e.g.: field engineering scheduling. 


\section{Digital Twins for Simulation Systems}

\section{System twins:}

Balancing the multiplicity ofkpi which balances the revenue cost and product lifecycle for e.g.: maintenance of Mega watt Bank.

\section{CUTTING EDGE}

\subsection{Technology}

Artificial history of the system can be accessed with simulation. The behavior of the system analyzed in a sophisticated manner with help of model. Model takes set of hypotheses concerning operation of the system Simulation very well known to engineering industry has been in use for several decades. Customized blocks in simulation system will help in depth analysis of problems and sub problems.in a typical engineering process most of the times results are misinterpreted, costly, time consuming, and existence of multiple simulation systems will mess up exact and accurate results. This makes there is need of digitizing the manufacturing industry. Aiding Realtime accessing of the system with simulation models which simplifies data processing and real view of the system with help of mental models will solves many problems latest industry.

Most of the simulation process are aspect based. Simulation application will solve only one aspect. Data maintenance is bottleneck in simulation system. Combining various domain simulation systems is unmanageable. Hence a new system is proposed to digitize manufacturing industry. These results of the simulation system will be not be aspect based and multiple perspectives needs to be considered. Many angels, parameters need to be considered. Various aspects in multiple dimensions will solve issues and challenges in the present simulation system. This can be achieved with creating mental models of the physical system. This is known as digital twin which digitizes the manufacturing industry. Digital twin refers to computerized companion of the physical assets that can be used for various purposes. The data from the sensors installed on the physical objects to represent real time status working condition or position. Digital twin uses 3D modelling to create companion of physical objects which provides way to project physical objects to digital world.

1. This system will give zero anomaly results with shot duration and less cost.

2. Recuse of data in different simulation systems can be achieved .

3. Swift turn around time for any breakage in the system.

4. Data analytics applied on simulation system for getting better results.

System (Digital Twin) used to find the status of actual physical object which provides way to project physical object to digital world. Making simulation system more powerful with real time sensor data changes outputs more accurate.different algorithms will be applied on simulation models which gives more control on the developed tools.

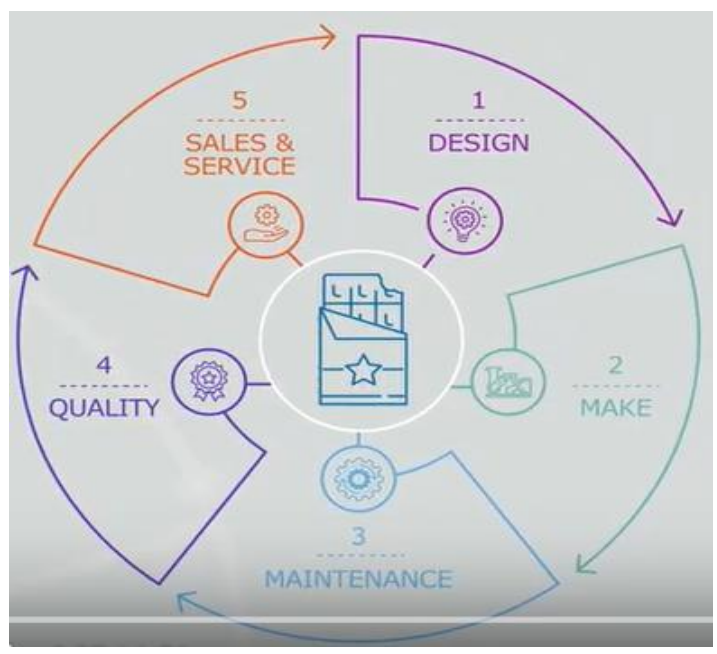

Fig 2:digital twin life cycle

Figure 2 represents the life cycle of digital twin which consists of five phases. They are Design, make, maintenance, quality and sales Services. It starts with design customer data like taste, flavor and color trends are analyzed by artificial intelligence and visualized by 3D Technology A distinct simulation is injected for measuring system performance. Various simulation systems with various algorithms are grouped for accurate results. In production process huge data will be generated it can be used for better management and control. This data is time stamp based. For utilizing this temporal data no need to own the production machine. Data can be collected by IoT Environment. Various scenarios are played with temporal data to analyze system performance. In maintenance stage future can be predicted with advanced analytics method and spare can be ordered with out any delay. Quality checks are indispensable with the new system. Patterns can be easily analysed. with connected architecture customers identification is easy hence improved sales and services.

Motive is using multiple aspects and parameters in a new system for sophisticated results which reuses various simulation models data, algorithms

Virtual Physical Systems have been proposed as a key concept of Industry 4.0 architectures.

field devices, machines, plants, factories and even individual products will increasingly be connected

to a network (e.g. the Internet or a private factory network). This will allow them to become searchable, explorable, and analyzable via the network, leading to potentially new types of services and business models in the value chain. However, this also leads to a significant increase of available online objects,

making it difficult to manage all data sources both from a computer system point of view as well as from a human interaction point of view.

On the computational side, Virtual Physical Systems have been proposed as a key concept of Industry 4.0 architectures. A Virtual Physical System can be described as a set of physical devices, objects and equipment's that interact with a virtual cyberspace through a communication network. In 
This model, each physical entity be a digital representation of the real entity, thus is sometimes called "Digital Twin". The Digital Twin can monitor and control the physical entity, while the physical entity can send data to update its virtual model.

\section{IMPLEMENTATION\& RESULTS}

In This Architecture data is retrieved from different data repositories using sensors, which implement the Digital Twin concept for UAV.figure 3 gives the idea of architecture of UAV virtual model.

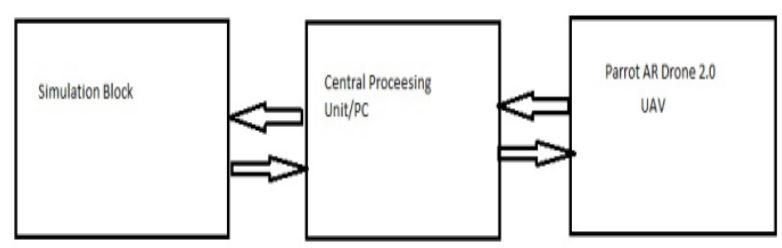

Figure3: Architecture of UAV Digital twin

Central processing unit or PC will have simulation models and direct connectivity with UAV.

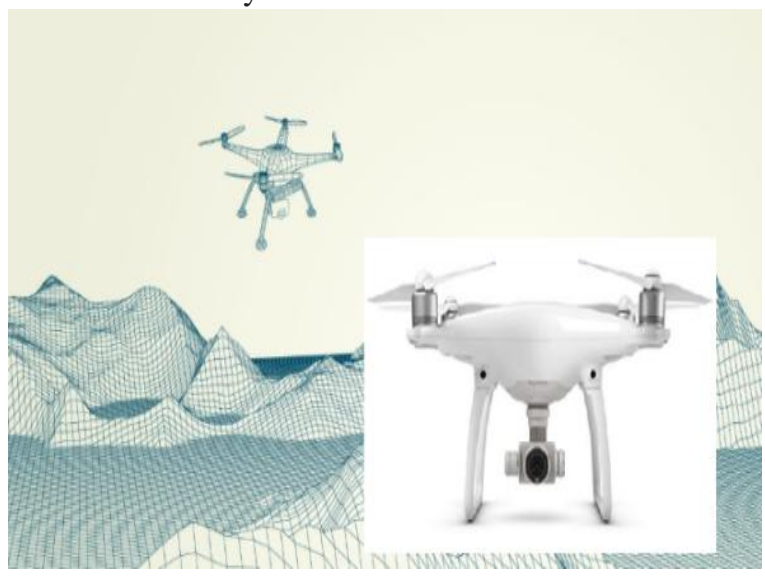

Figure 4: Digital Twin of Quadcoptor/UAV

Figure 4 represents the digital twin of quadcopter.In digital twin simulation and realtime device data will be accessed, simulated, analysed and fixed issues on the fly.this is the greatest advantage of digital twin concept.simulink is used for simulating the UAV and at the same time realising ,interpreting the UAV realtime data. With this precise, faulty location/device will be identified and necessary preventive actions can be taken, which indirectly reduces the turn around time of a UAV and maintenance cost of UAV will be reduced significantly and also the down time of the UAV will be reduced in a significant manner.

\section{SIMULATION}

In order to validate the digital twin concept, the system was simulated using a Matlab Simulink Model. For Digital Twin we have used Parrot AR Drone 2.0. The state space representation of the ARDrone's Platform dynamics are taken from ARDrone Simulink Development Kit [7].Figure 3 RepresentshighlevelArchitechure of UAV Digital Twin. Firstly Virtual Replica model will be created in MATLAB as shown in Figure 5.

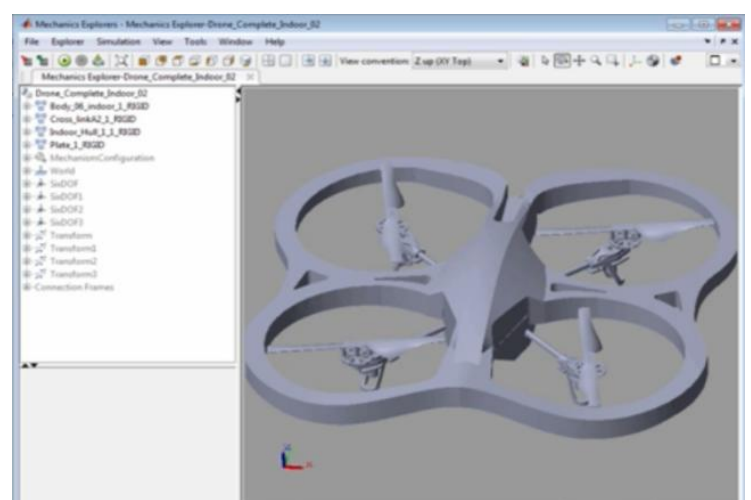

Figure 5: Indoor Model of Parrot AR Drone 2.0

4.1 Simulink Block :

The desired path that the drone is to take outlined in Table 4.1

\begin{tabular}{|c|c|c|}
\hline Waypoint & $\begin{array}{c}\mathbf{X} \\
\text { Coordinate(m) }\end{array}$ & $\begin{array}{c}\text { Y } \\
\text { Coordinate(m) }\end{array}$ \\
\hline 1 & 2.5 & -1 \\
\hline 2 & 2.5 & 1 \\
\hline 3 & -2.5 & 1 \\
\hline 4 & -2.5 & -1 \\
\hline
\end{tabular}

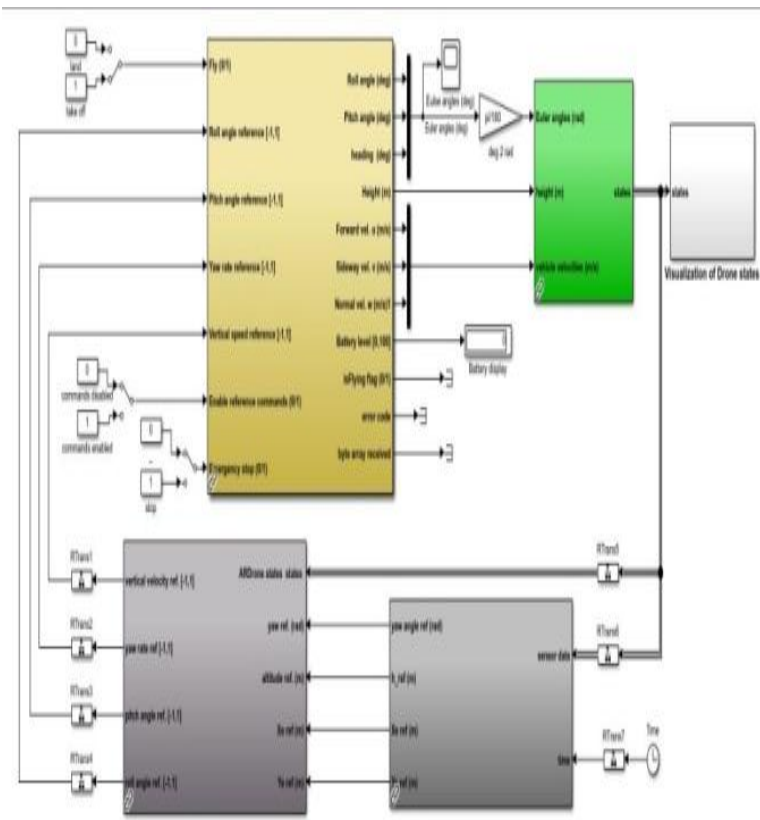

Figure 6:Simulink Blocks of Parrot ARDrone 2.0 for waypoints

Figure 6 : The Simulink model used includes a state space representation from the AR Drone Simulink Development Kit[7].

\subsection{Simulation Results :}

AR drone is simulated and resulting height, roll,yaw,pitch are plotted in Figure 7 


\section{Digital Twins for Simulation Systems}

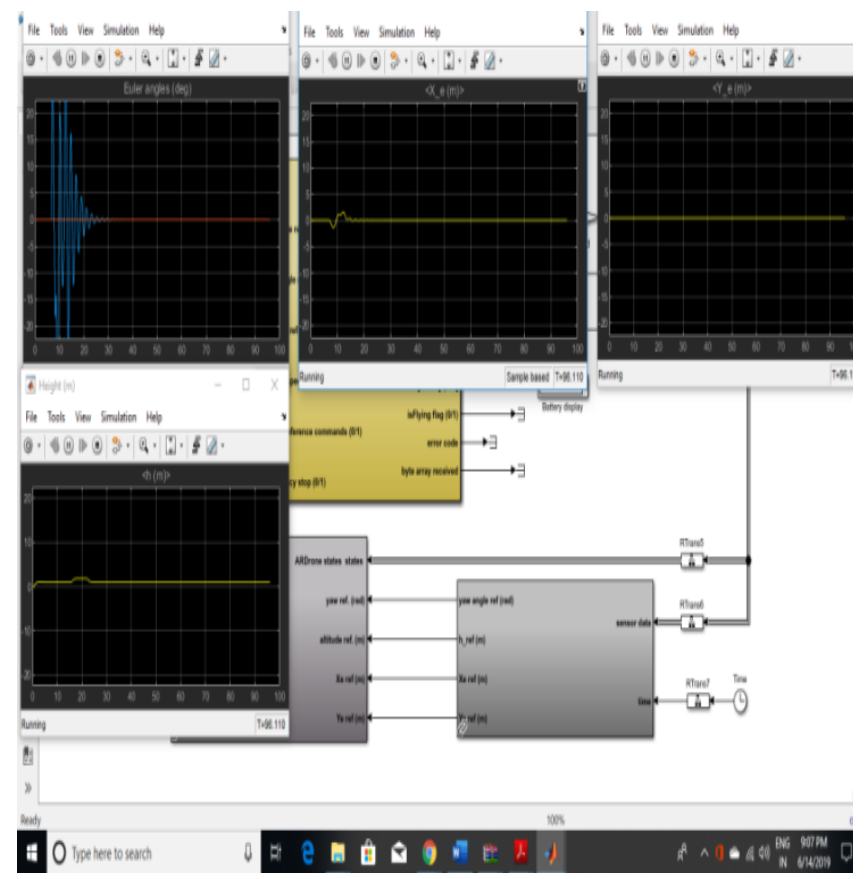

Figure 7:results of virtual model of UAV waypoints

\subsection{Real time device Results :}

After simulation, With Parrot AR Drone 2.0 Wifi network the real time device can be accessed.and performance of UAV is interpreted. Figure 8 : Shows Realtime accessing of parrot Ar Drone 2.0 and outputs.

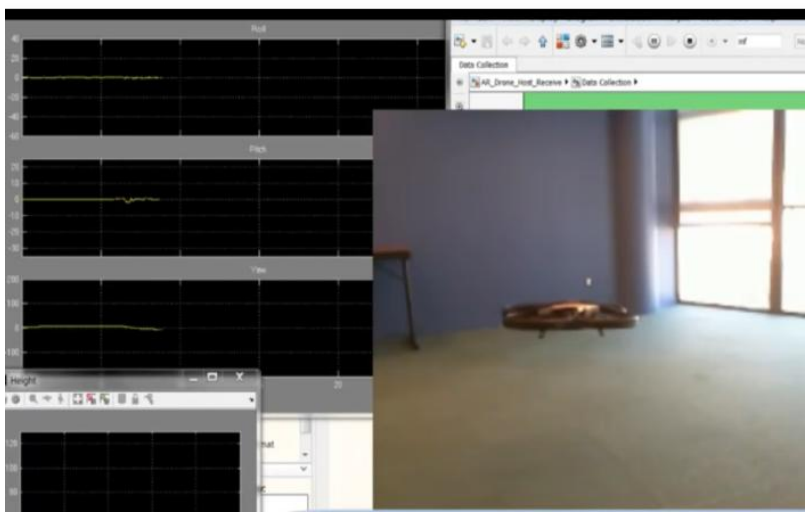

Figure 8:results of real UAV waypoints

\section{CONCLUSION AND FUTURE WORK}

A Digital Twin (mostly software) will run in parallel to the actual equipment.Predictive Analytics can be achieved on Digital Twins much ahead of time before detectable conditions are encountered in the real equipment.Reduced unplanned downtime -improved operational, maintenance costs.High Fidelity Simulation Systems for Testing \& Training with reduced costs.

In this work, the concept of the Digital Twin is demonstrated with UAV (AR Drone)

Presently predictive monitoring and diagnostics of a aircraft is done by various predictive monitoring algorithms. With Data analytics and machine learning algorithms monitoring of UAV can be done for a better performance. New PTMD systems for UAV need to be developed.

\section{REFERENCES}

1. MATLAB/Simulink. www.mathworks.de/products/simulink/
2. Banks, J. Discrete-Event System Simulation, Prentice-Hall: 2010

3. 3. S. Kadry; A. El Hami (Eds.) E-Systems for the 21s Century: Concept, Developments, and Applications. Apple Academic Press, 2015

4. B. Srivastava and J. Koehler, "Web service composition-current solutions and open problems," in ICAPS 2003 workshop on Planning for WebServices, vol. 35,2003 , pp. 28-35.

5. Simmechanics. www.mathworks.de/products/simmechanics/

6. Schlette, C., et al. "Virtual commissioning of automated micro-optical assembly." SPIE LASE. International Society for Optics and Photonics, 2015.

7. D.E SanabriaArdrone Simulink development kit v 1.1 [On-line]. Available

http://www.mathworks.com/matlabcentral/fileexchange/4 3719-ar-drone-simulink-development -kit-v1-1

8. Motion Analysis systems . [Online] . Available http://www.motionanalysis.com

9. S.J Mills , J. J Ford, and L. Mejias “ Vision Based control for fixed wing UAVS inspecting locally linear infrastructure using skid-to-turn maneuvers ", Journal of Intellegent and Robotic Systems vol.61, no- 1-4, pp 29-42, 2011

10. T. Roth, E. Song, M. Burns, H. Neema, W. Emfinger and J. Sztipanovits, "Cyber-physical system development environment for energy applications," in ASME 11th International Conference onEnergy Sustainability, Charlotte, NC, 2017. 